\title{
Protection of Oligodendrocytes Through Neuronal Overexpression of the Small GTPase Ras in Hyperoxia- Induced Neonatal Brain Injury
}

\author{
Meray Serdar ${ }^{1}$, Josephine Herz ${ }^{1}$, Karina Kempe ${ }^{1}$, Elke Winterhager' ${ }^{2}$, Holger Jastrow ${ }^{2,3}$, \\ Rolf Heumann ${ }^{4}$, Ursula Felderhoff-Müser ${ }^{1 *}$ and Ivo Bendix ${ }^{1 *}$
}

'Department of Pediatrics I, Neonatology, University Hospital, University Duisburg-Essen, Essen, Germany, ${ }^{2}$ Imaging Center Essen, EM Unit, University Hospital Essen, University Duisburg-Essen, Essen, Germany, ${ }^{3}$ Institute of Anatomy, University Hospital Essen, University Duisburg-Essen, Essen, Germany, ${ }^{4}$ Biochemistry II, Molecular Neurobiochemistry, Faculty of Chemistry and Biochemistry, Ruhr University Bochum, Bochum, Germany

Prematurely born infants are highly susceptible to various environmental factors, such as inflammation, drug exposure, and also high environmental oxygen concentrations.

OPEN ACCESS

Edited by:

Olivier Baud,

Geneva University Hospitals (HUG), Switzerland

Reviewed by:

Susan Cohen,

Medical College of Wisconsin,

United States

Wei-Liang Chen

Medical College of Wisconsin,

United States

*Correspondence:

Ursula Felderhoff-Müser ursula.felderhoff@uk-essen.de; Ivo Bendix

ivo.bendix@uk-essen.de

Specialty section: This article was submitted to Pediatric Neurology, a section of the journal Frontiers in Neurology

Received: 08 December 2017 Accepted: 06 March 2018 Published: 21 March 2018

Citation:

Serdar M, Herz J, Kempe K,

Winterhager $E$, Jastrow $H$, Heumann R, Felderhoff-Müser $U$ and

Bendix I (2018) Protection of Oligodendrocytes Through Neuronal Overexpression of the Small GTPase Ras in Hyperoxia-Induced Neonatal Brain Injury.

Front. Neurol. 9:175 doi: 10.3389/fneur.2018.00175
Hyperoxia induces perinatal brain injury affecting white and gray matter development. It is well known that mitogen-activated protein kinase signaling is involved in cell survival, proliferation, and differentiation. Therefore, we aim to elucidate cell-specific responses of neuronal overexpression of the small GTPase Ras on hyperoxia-mediated brain injury. Six-day-old (P6) synRas mice (neuronal Ras overexpression under the synapsin promoter) or wild-type littermates were kept under hyperoxia (80\% oxygen) or room air (21\% oxygen) for $24 \mathrm{~h}$. Apoptosis was analyzed by Western blot of cleaved Caspase-3 and neuronal and oligodendrocyte degeneration via immunohistochemistry. Short-term differentiation capacity of oligodendrocytes was assessed by quantification of myelin basic protein expression at P11. Long-lasting changes of hyperoxia-induced alteration of myelin structures were evaluated via transmission electron microscopy in young adult animals (P42). Western blot analysis of active Caspase-3 demonstrates a significant upregulation in wild-type littermates exposed to hyperoxia whereas synRas mice did not show any marked alteration of cleaved Caspase-3 protein levels. Immunohistochemistry revealed a protective effect of neuronal Ras overexpression on neuron and oligodendrocyte survival. Hyperoxia-induced hypomyelination in wild-type littermates was restored in synRas mice. These short-term protective effects through promotion of neuronal survival translated into long-lasting improvement of ultrastructural alterations of myelin sheaths in mice with neuronal overexpression of Ras compared with hyperoxic wild-type mice. Our data suggest that transgenic increase of neuronal Ras activity in the immature brain results in secondary protection of oligodendrocytes from hyperoxia-induced white matter brain injury.

Keywords: preterm birth, brain injury, hyperoxia, neuronal Ras, white matter injury, neuroprotection

\section{INTRODUCTION}

Within the last decade, mortality of very preterm infants and critically ill-term born infants has decreased by $25 \%$ due to major advances in obstetrics and neonatal intensive care (1). However, perinatal brain injury is still a leading cause of death and disability in children (2). With approximately $5.5-11.4 \%$ of all live births, the number of prematurely born infants has increased in industrialized 
countries (3), which may further rise due to increasing infertility treatments, multiple pregnancies, and higher maternal age (4). Due to considerable progress in perinatal management of highrisk infants, long-term survival has become an almost expected outcome. However, even in the absence of severe intracranial pathology such as intraventricular hemorrhage or periventricular leukomalacia affecting $10 \%$ of very preterm born children (5), diffuse white matter (WM) injury and reduction of cortical gray matter volume are observed in most survivors. This is often associated with cognitive impairment and behavioral problems such as attention deficit disorder, autism, and development of psychiatric disease in later life (6-9).

Compared with intrauterine conditions, preterm infants suffer from chronic or fluctuating exposure to supra-physiological oxygen levels involved in prematurity-related diseases such as retinopathy of prematurity and bronchopulmonary dysplasia $(10,11)$. However, there is mounting evidence from clinical and experimental studies that large amounts of oxygen in the neonatal period also disturb brain maturation involving oligodendrocyte degeneration and impaired differentiation leading to hypomyelination (12-14). Importantly, these short-term alterations of myelination translate into long-lasting structural WM alterations (15-19) associated with hyperactivity and coordination deficits at adolescent age (20), and cognitive impairment persisting into adulthood $(19,21)$.

Several cellular mechanisms of hyperoxia-induced preterm brain injury including apoptosis, autophagy, differences in modulation of reactive oxygen species, and inflammation have been identified $(17,21-26)$. At the molecular level, our previous work demonstrated the importance of the mitogen-activated protein kinase (MAPK) signaling pathway $(22,27)$. It was shown that hyperoxia reduces Ras GTPase activity and downstream signaling pathways (e.g., phosphorylation of the extracellular regulated kinase (ERK)) (27), which modulate important cellular responses such as differentiation and survival (28). Of note, using synRas-transgenic mice overexpressing constitutively activated Ras (29) in the neuronal compartment, we confirmed the important involvement of this pathway demonstrating protection against oxygen-induced cell death (27). However, long-term consequences for the development of WM injury and direct effects on hyperoxia-induced oligodendrocyte degeneration and hypomyelination are still unknown. Moreover, neuronal Ras activation in adult mice leads to an increase of dendritic spine density and synapses as well as increased neuronal activity (30). Taking into account that oligodendrocyte development is not only controlled intrinsically, e.g., by transcription factors but also by neuronal activity (31-33), modulation of neuronal activity and signaling pathways may determine oligodendrocyte responses and subsequent effects on myelination in oxygen-related injury of the immature brain.

Although our understanding of consequences of neonatal hyperoxia on WM development significantly improved within the last decade, the impact of a potential cross talk between the neuronal compartment and myelinating oligodendrocytes is still unknown. Given the important role of MAPK signaling in oxygen-induced preterm brain injury and the fact that neuronal activity modulates oligodendrocyte development, we aimed to elucidate the impact of a transgenic overexpression of constitutively activated Ras in neurons on hyperoxia-induced oligodendrocyte degeneration, myelination, and long-lasting ultrastructural alterations of the WM.

\section{MATERIALS AND METHODS}

\section{Animals and Housing}

Transgenic mice overexpressing the mutated human-Ras gene [Ha-Ras G12V (exchange of glycin through valin)] under the synapsin 1 promoter (synRas) were used. These mice reveal a constitutive activation of Ras in post-mitotic neurons starting at postnatal day $4(\mathrm{P} 4)$. Transgenic males were mated with wild-type female C57BL/6 mice. Genotyping was performed by PCR as described previously (29). The following primers were used to identify the mutated human transgene G12V (360 bp); Fwd (synRas for) AAT TCC AGC TGA GCG CCG GT and Rev (synRas rev) GAC ACA CTC ATG AGA TGC CT. Animals were housed under 12-h light/dark cycle, food and water ad libitum. All animal experiments were approved and performed in accordance with the guidelines of the University Hospital Essen, Germany and with local government approval by the State Agency for Nature, Environment and Consumer Protection North Rhine-Westphalia.

\section{Experimental Procedures}

Due to the well-known delay in the brain growth spurt period, which corresponds to the most susceptible period of brain injury in neonatal rodents (34-36), 6-day-old mice were used. SynRas pups and their appropriate wild-type littermates were placed in an oxygen chamber containing $80 \%$ oxygen (OxyCycler, BioSpherix, USA) for $24 \mathrm{~h}(\mathrm{HO})$ together with their lactating dams. Control pups were kept under normoxic conditions (21\% oxygen, NO) resulting in four study groups: wild-type hyperoxia (BL6 HO); synRas hyperoxia (synRas HO); wild-type normoxia (BL6 NO); and synRas normoxia (synRas NO). Pups were sacrificed at P7, $\mathrm{P} 11$, and P42 under deep anesthesia. In accordance with our previous studies $(17,21,27,37), 24 \mathrm{~h}$ is sufficient to modulate myelin basic protein (MBP) expression and acute cellular degeneration determined at P11 and P7, respectively, without an induction of acute injury to the dams $(27,37)$. Since differences in MBP expression following neonatal hyperoxia are less prominent few days after the peak of brain growth spurt period (P7-P10) initial myelination was assessed at term-equivalent age (P11) $(34,36)$ according to our previous work $(15,17,19,21$, 37). Ultrastructural alterations of myelination were analyzed in young adult mice at $\mathrm{P} 42$ by transmission electron microscopy as described below. For protein analysis, mice were transcardially perfused with PBS and brain hemispheres were snap frozen in liquid nitrogen. For histological studies, pups were transcardially perfused with PBS followed by $4 \%$ paraformaldehyde (PFA, Sigma-Aldrich). Brains were postfixed in 4\% PFA overnight at $4^{\circ} \mathrm{C}$ and embedded in paraffin. Based on our previous experience $(17,21)$, whole hemispheres (excluding cerebellum) were used for Western blot analysis of MBP and cleaved Caspase-3 expression. 


\section{Immunoblotting}

Western blotting was performed as described previously $(17,21)$, with adaptions in epitope detection. Membranes were blocked with $5 \%$ non-fat dry milk in Tris-buffered saline, $0.1 \%$ Tween-20 (TBST, Sigma Aldrich) and incubated overnight $\left(4^{\circ} \mathrm{C}\right)$ with the following primary antibodies: polyclonal rabbit anti cleaved Caspase-3 (1:1,000, Cell Signalling, Germany), monoclonal mouse anti-MBP antibody (1:10,000, Abcam, UK), monoclonal mouse anti-Olig2 (1:1000, Millipore, Germany), and polyclonal rabbit anti-glyceraldehyde 3-phosphate dehydrogenase antibody (1:1,000, Santa Cruz, Germany). Horseradish peroxidase-conjugated secondary antimouse $(1: 5,000)$ or anti-rabbit $(1: 2,000$, both DAKO, Denmark) antibodies were used. All antibodies were diluted in 5\% non-fat dry milk in TBST. Antibody binding was detected by using enhanced chemiluminescence (GE Healthcare Life Sciences, Germany). For visualization and densitometric analysis, ChemiDoc ${ }^{\mathrm{TM}} \mathrm{XRS}+$ imaging system and ImageLab software (Bio-Rad, Germany) were used. Representative images of full-length Western blot are depicted in Figure S1 in Supplementary Material.

\section{Immunohistochemistry and Confocal Microscopy}

After deparaffinization, $10 \mu \mathrm{m}$ coronal sections $(-2.255 \pm 0.6 \mathrm{~mm}$ from bregma) were rehydrated. Antigen retrieval was performed in a preheated $10 \mathrm{mM}$ sodium citrate buffer $(\mathrm{pH}$ 6.0) for $30 \mathrm{~min}$. After blocking with $1 \%$ bovine serum albumin, $0.3 \%$ cold fish skin gelatine in $0.1 \%$ Tween-20 TBS (all Sigma-Aldrich) slides was incubated with primary antibodies overnight at $4^{\circ} \mathrm{C}$ followed by appropriate secondary antibody incubation for $1 \mathrm{~h}$ at room temperature. Sections were counterstained with 4,6-diamidino2-phenylindole (DAPI) (1 $\mu \mathrm{g} / \mathrm{ml}$, Invitrogen, Germany). Myelination was evaluated at P11 using the primary mouse anti-rat MBP antibody (1:100, SMI-99, Sternberger Monoclonals, USA). Degeneration of oligodendrocytes or neurons were detected at P7 via co-labeling of Olig2 (1:100, monoclonal mouse antiOlig2, Millipore) or NeuN (1:200, polyclonal rabbit anti-NeuN, Millipore) with terminal deoxynucleotidyl transferase mediated biotinylated dUTP nick end labeling (TUNEL, In Situ cell death detection kit, FITC; Roche, Germany), performed according to the manufacturers' instructions. All primary antibodies were followed by appropriate secondary antibody staining (anti-mouse Alexa Fluor 488, anti-rabbit Alexa Fluor 555, anti-mouse Alexa Fluor 555; all 1:500, Invitrogen). Stained brain sections were analyzed by confocal microscopy (A1plus, Eclipse Ti, with NIS Elements AR software, Nikon, Germany) using $10 \times$ or $20 \times$ objectives. Three laser lines (laser diode: $405 \mathrm{~nm}$; Ar laser: $514 \mathrm{~nm}$; G-HeNe laser: $543 \mathrm{~nm}$ ) and three different filters (450/50-405 LP, 515/20-540 LP, 585/65-640 LP) were used for image acquisition. Confocal $z$-stacks of $10 \mu \mathrm{m}$ thickness $(z$-plane distance $3 \mu \mathrm{m})$ were converted into two-dimensional images using maximum intensity projections. Image acquisition and analysis were performed by an observer blinded to treatment and genotype. The quantification of each staining was performed with the NIS Elements AR software. For MBP stained sections, large scale images (stitching) of deep cortical WM were generated. Degenerating oligodendrocytes or neurons were quantified by counting triple positive cells (DAPI,
TUNEL, Olig2/NeuN) in eight bilateral regions of interest (ROI) derived from two sections per animal (each ROI: $396,900 \mu \mathrm{m}^{2}$, retrosplenial and primary somatosensory cortex; cingulum, deep cortical WM; parafascicular nucleus and posterior complex of the thalamus; CA1 and CA2 of the hippocampus).

\section{Electron Microscopy}

Six-week-old transgenic synRas mice and their appropriate wildtype littermates exposed to $24 \mathrm{~h}$ hyperoxia at P6 were perfused with $37^{\circ} \mathrm{C}$ warm phosphate buffer solution followed by $2.5 \%$ glutaraldehyde in $0.1 \mathrm{M}$ cacodylate buffer (CB). Brains were removed and immediately transferred into 12 -well plates filled with $2.5 \%$ glutaraldehyde in $0.1 \mathrm{M} \mathrm{CB}$. Brains were cut into $1 \mathrm{~mm}$ thick frontal slices for better penetration. One section clearly showing the corpus callosum was chosen from each specimen and trimmed in a way that the latter was preserved in a final $\sim 1 \mathrm{~mm} \times 1 \mathrm{~mm} \times 1 \mathrm{~mm}$ tissue block. After fixation lasting for at least $12 \mathrm{~h}$, blocks were washed in $\mathrm{CB}$ at room temperature for $3 \times$ $30 \mathrm{~min}$, followed by $1 \%$ osmium tetroxide (PolyScience, USA) in $\mathrm{CB}$ for $3.5 \mathrm{~h}$ in the dark. After washing in CB, 30 and 50\% ethanol (45 min each) was added followed by incubation in 1\% uranylacetate (Electron Microscopy Science, USA) in 70\% ethanol in the dark for $1 \mathrm{~h}$. Blocks were further incubated in 80,90 , and $96 \%$ ethanol (45 min each) and 100\% ethanol $(3 \times 20 \mathrm{~min})$ followed by propylene oxide $\left(2 \times 20 \mathrm{~min}\right.$, Sigma) and $\mathrm{EPON}^{\circledR}$ (Sigma) solutions in propylene oxide with increasing $\mathrm{EPON}^{\circledR}$ concentration (3:1, 1:1, 1:3; 60 min each, 100\% EPON ${ }^{\circledR}$ overnight) at room temperature. Polymerization was performed at $60^{\circ} \mathrm{C}$ for 2 days. After trimming solid EPON ${ }^{\circledR}$ blocks were cut on a Reichert-Jung Ultracut $^{\circledR}$ E ultramicrotome (Reichert AG, Austria) set to a thickness of $50 \mathrm{~nm}$. Sections were mounted on 200 mesh hexagonal copper grids and treated with $1 \%$ uranylacetate for $4 \mathrm{~min}$ and lead citrate trihydrate $\left(0.4 \%\right.$ in $\left.\mathrm{ddH}_{2} \mathrm{O}\right)$ for $3 \mathrm{~min}$ for contrast enhancement. A Zeiss transmission electron microscope (EM 902A; Zeiss, Germany) was used for final investigation at $80 \mathrm{kV}$ at magnifications from $3,000 \times$ to $100,000 \times$. Digital image acquisition was performed by a Mega View III slow-scan-CCD camera connected to a PC running ITEM $^{\circledR} 5.0$ software (Soft Imaging Systems, Germany); images were saved as uncompressed 16 bit TIFF files and further processed using Adobe ${ }^{\circledR}$ Photoshop $^{\circledR}$ CS5. Alterations of the myelin sheath were analyzed by using the Image J software (Java Software).

To evaluate myelin deficits a total of 1,645 axons (BL6 HO) and 1,488 axons (synRas HO) in 60-65 fields of view at 10,000x magnification $\left(61.44 \mu \mathrm{m}^{2}\right.$ each) derived from 4 mice per group were analyzed. The percentage of non-myelinated axons related to the total number of axons per field of view was quantified. Pathological alterations in the myelin sheath of myelinated axons were evaluated through quantification of the number of axons with myelin encapsulation and decompaction, and axons with increased adaxonal space. Only axons whose contour was completely within each photograph were used. Analysis was carried out by an individual blinded to group assignment.

\section{Statistical Analysis}

Data are presented as mean + SD. For statistical analysis, the GraphPad Prism 6.0 software package (GraphPad Software, 
USA) was used. After checking for normal distribution using D’Agostino-Pearson omnibus test either ordinal one-way analysis of variance with post hoc Bonferroni multiple comparisons test or Kruskal-Wallis with Dunn's multiple comparisons test were applied (Figures 1-3). For comparison of two groups (Figure 4) either Student's $t$-test (parametric) or in case of non-parametric data Mann-Whitney $U$ test was used. $p$-Values less than 0.05 were considered as statistically significant.

\section{RESULTS}

\section{Neonatal Hyperoxia-Induced Apoptosis Is Decreased by Neuronal Ras Activation}

To evaluate the extent of brain injury following neonatal hyperoxia at P6, we determined cleaved Caspase-3 protein abundance immediately after $24 \mathrm{~h}$ hyperoxia. In line with previous findings (27), we detected a significant increase of cleaved Caspase-3 levels in protein lysates of wild-type mice following hyperoxia (BL/6 HO) compared with normoxic controls (BL/6 HO) and compared with synRas mice exposed to hyperoxia (synRas $\mathrm{HO}$ ) (Figure 1). No alteration of cleaved Caspase-3 protein levels was determined in synRas animals at normoxia (synRas NO, Figure 1).

\section{Constitutive Neuronal Ras Activity Ameliorates Neuronal and Oligodendrocyte Degeneration in Neonatal Mice Following Hyperoxia}

To investigate the effect of hyperoxia and neuronal Ras overexpression at a cellular level, indicated by TUNEL labeling, we analyzed the amount of degenerating neurons (Figure 2A) and oligodendrocytes (Figure 2B). Hyperoxia significantly increased

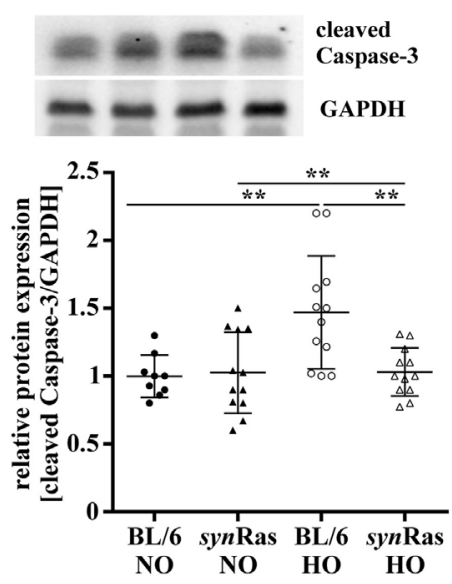

FIGURE 1 | Apoptosis caused by hyperoxia is reduced in transgenic synRas mice. Induction of apoptosis was determined by relative protein expression analysis for cleaved Caspase-3 by Western blot in lysates from hemispheres of postnatal day 7 (P7) mice that were exposed to either normoxia [21\% oxygen (NO)] or hyperoxia [24 h, 80\% oxygen $(\mathrm{HO})$ ] at P6 in synRas mice or their respective wild-type littermates (BL/6). Data are represented as relative protein expression [cleaved Caspase-3/glyceraldehyde 3-phosphate dehydrogenase $(\mathrm{GAPDH})]$ normalized to the control group (BL/6 NO) $\left.{ }^{* \star} p<0.01\right)$. neuronal degeneration in cortical, hippocampal, and thalamic (TH) regions of wild-type mice, which was significantly reduced in synRas-transgenic mice (Figure 2A). For oligodendrocyte degeneration, we detected a similar increase in BL/6 wild-type mice (Figure 2B). Importantly, selective neuronal activation of Ras led to a significant decrease of oligodendrocyte degeneration in TH and WM structures (Figure 2B).

\section{Hyperoxia-Induced Hypomyelination Is Prevented in synRas Mice}

A major hallmark of oxygen-induced toxicity to the immature brain is characterized by hypomyelination $(15,19,21)$. To clarify whether the unexpected decrease in oligodendrocyte degeneration in syn Ras mice translates into disturbed myelination, we next examined MBP expression at P11 after exposure to hyperoxia at P6. As shown in representative images in Figure 3A, hyperoxia resulted in a decrease of MBP expression in wild-type BL/6 mice which was restored in $s y n$ Ras mice (Figure 3A). Quantification of protein lysates by Western blot analysis isolated from whole hemispheres confirmed these qualitative observations and revealed significantly reduced levels of MBP expression in animals exposed to hyperoxia compared with normoxic control wild-type control mice (Figure 3B). Normoxic and hyperoxic synRas mice revealed similar levels of MBP expression compared
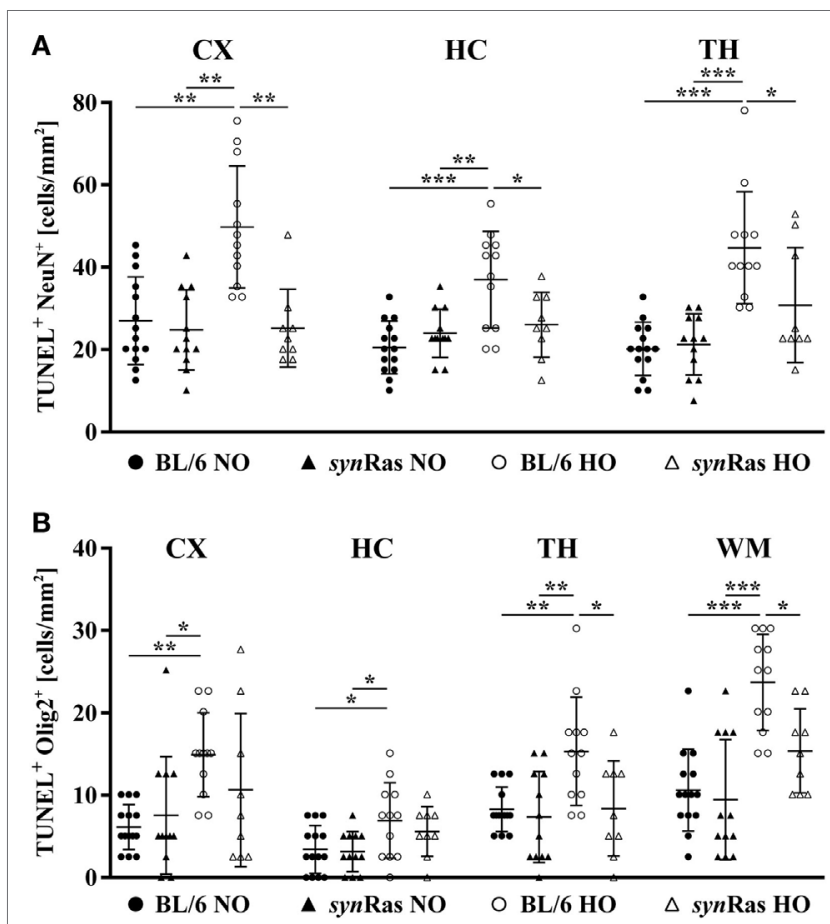

FIGURE 2 | Hyperoxia-induced neuronal and oligodendrocyte degeneration is ameliorated in synRas mice. Brain sections from P7 mice either exposed to normoxia [21\% oxygen (NO)] or hyperoxia [24 h, 80\% oxygen $(\mathrm{HO})$ ] at P6 in synRas mice or their respective wild-type littermates (BL/6) were analyzed. Neuronal (NeuN) (A) and oligodendrocyte (Olig2) (B) degeneration was determined in cortical $(\mathrm{CX})$, hippocampal $(\mathrm{HC})$, thalamic $(\mathrm{TH})$, and white matter (WM) regions by double-labeling with TUNEL $\left({ }^{\star} p<0.05,{ }^{* *} p<0.01\right.$, and $\left.{ }^{\star \star \star} p<0.001\right)$ 


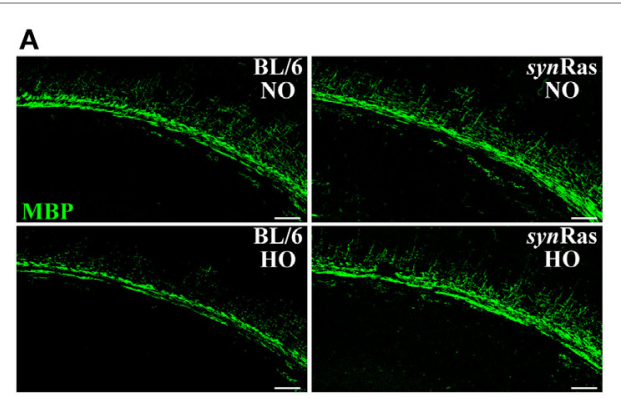

B

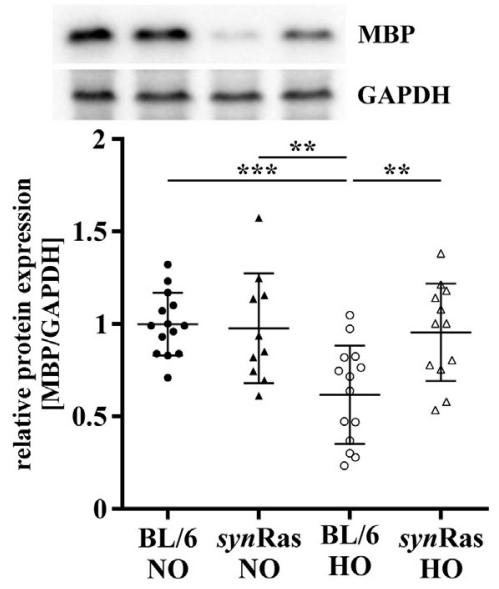

C

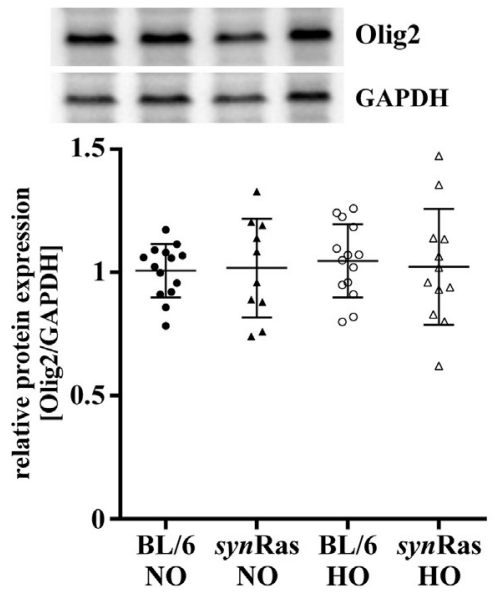

FIGURE 3 | Impact of neuronal Ras activation in synRas on short-term hyperoxia-induced hypomyelination. Myelin basic protein (MBP) expression was analyzed 4 days post hyperoxia. (A) Representative

immunohistochemical staining of the deep cortical white matter assessed by confocal microscopy (scale bar $=100 \mu \mathrm{m}$ ). (B) MBP and (C) Olig2 protein expression was analyzed by Western blot in protein lysates of hemispheres of P11 mice that were exposed to either normoxia [21\% oxygen (NO)] or hyperoxia [24 h, 80\% oxygen (HO)] at P6 in synRas mice or their respective wild-type littermates (BL/6). Data are represented as relative protein expression [MBP/glyceraldehyde 3-phosphate dehydrogenase (GAPDH) and Olig2/GAPDH] normalized to the control group (BL/6 NO) ${ }^{* \star} p<0.01$ and $\left.{ }^{\star \star \star} p<0.001\right)$.

with normoxic BL/6 controls (Figure 3B). Of note, 4 days after hyperoxia Olig2 expression was not significantly altered across all experimental groups (Figure 3C).

\section{Neuronal Ras Activation Protects From Hyperoxia-Induced Long-Lasting Ultrastructural Myelination Abnormalities}

Since our major focus of this study was to determine the impact of enhanced neuronal Ras activity in the context of hyperoxiainduced WM pathology and according to the fact that we did not observe any differences between normoxic control animals (BL/6 and synRas NO) across all previous analyses, we focused our analysis regarding long-term ultrastructural changes via electron microscopy to hyperoxic animals only. Assessing crosssections of the corpus callosum of 6-week-old mice after neonatal hyperoxia, we observed several myelin abnormalities including non-myelinated axons, axons with an increased adaxonal space as well as split sheaths with decompaction, myelin lamellae partially broken down into vesicular structures at the innermost region of the myelin sheath (internal loops) as well as focal myelin damage (Figure 4A). Comparing both injury groups, we detected a significant reduction regarding non-myelinated axons (Figure 4B), axons with an increased adaxonal space (Figure 4C), as well axons with signs of myelin encapsulation and decompaction (Figure 4D) in synRas following neonatal hyperoxia at P6.

\section{DISCUSSION}

Oxygen-induced preterm birth-related brain injury is associated with subtle neurodegeneration and impaired WM development resulting in long-lasting decrease of fractional anisotropy and diffusivity in the WM associated with neurodevelopmental disturbances $(17,19-21,38,39)$. Myelination is the key for efficient nerve conduction velocity proposed to strengthen circuitry throughout the nervous system (40). However, recent evidence also suggests a substantial impact of neuronal activity on myelination $(31,33)$. Our results demonstrate that the selective neuronal activation of the small GTPase Ras protects against hyperoxia-mediated neuronal but also oligodendrocyte degeneration and hypomyelination. Importantly, these short-term effects translated into longlasting improvement of myelin integrity resulting in a reduced amount of non-myelinated axons, an increase in axon-lamination, and an elevated number of axons with compact myelin.

Small GTPases from the Ras family are highly evolutionary conserved, molecular switches involved in important cellular responses such as proliferation, differentiation, and survival (28). We previously showed that hyperoxia reduces Ras activity and that transgenic mice overexpressing Ras under the synapsin 1 promoter (synRas) to drive the selective overexpression of V12-Ha-Ras in post-mitotic neurons (29) are protected against hyperoxia-induced cellular degeneration (27). Nevertheless, the cellular target of protection remained unclear. In this study, we show that cell-specific neuronal modulation of Ras protects neurons from cellular degeneration. According to our previous work demonstrating that hyperoxia-induced cell death is associated with reduced expression Ras effector molecules promoting cell survival (e.g., ERK1/2) (27), results of this study can be most likely explained by increased Ras-mediated neuronal survival signaling in synRas mice. Our results of regional neuronal cell death analysis correspond to previous studies revealing increased cell death 
A BL/6 HO
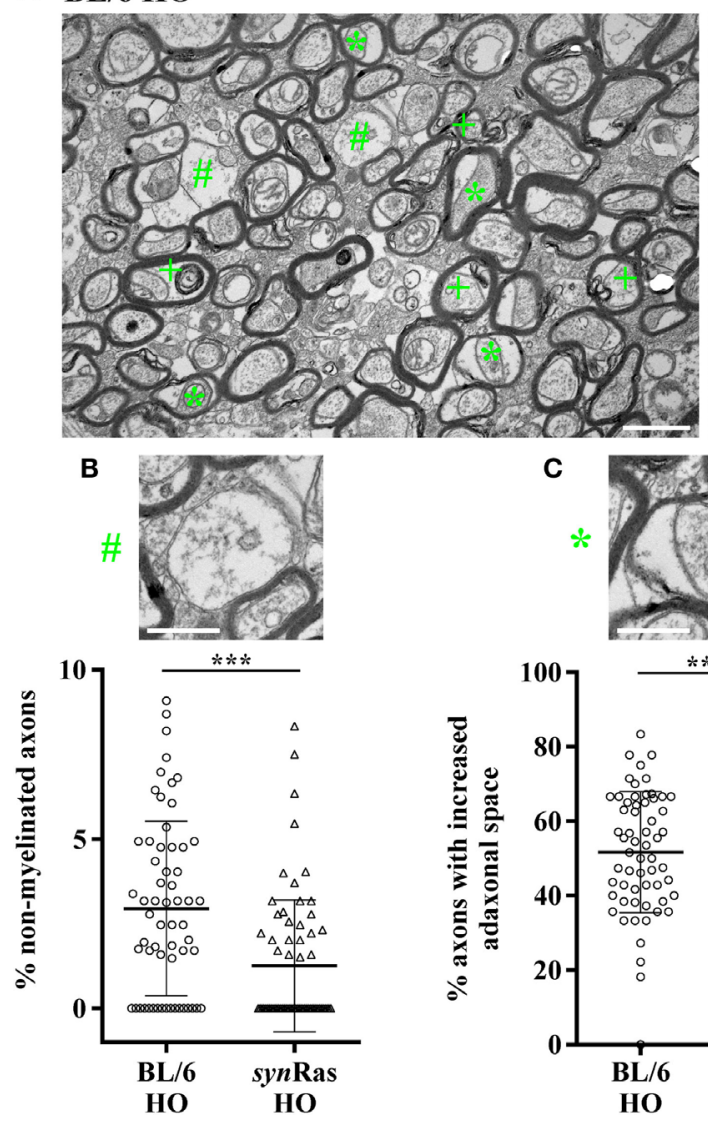

c

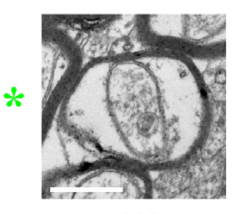

syn Ras HO

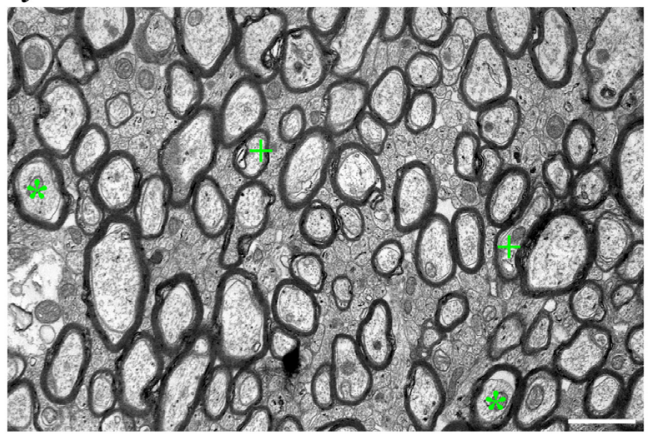

D

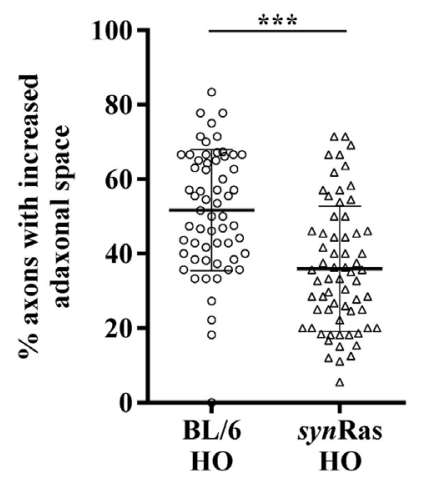

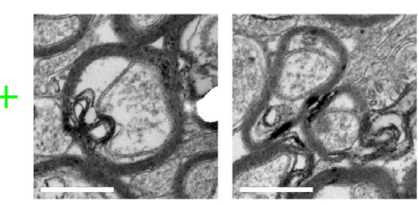

$*$

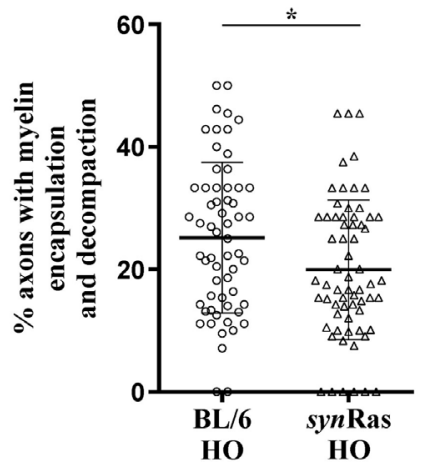

FIGURE 4 | Long-lasting ultrastructural changes of myelination in hyperoxic animals are diminished in transgenic synRas mice. (A) Representative electron microscopy images of young adult (P42) BL/6 and synRas mice either exposed to normoxia [21\% oxygen (NO)] or hyperoxia [24 h, 80\% oxygen (HO)] at P6. Quantification of non-myelinated axons [(B), \#], increased adaxonal space [(C), *], and axons with encapsulated and decompacted myelin sheets [(D), +]. Alterations are exemplified in panel (A). Scale bar in panel (A) is 1 and $0.5 \mu \mathrm{m}$ in higher magnified images in panels (B-D). $n=60-65$ analyzed field of views (61.44 $\mu m^{2}$ each) derived from four mice per group $\left({ }^{*} p<0.05\right.$ and $\left.{ }^{* \star *} p<0.001\right)$.

in the cortex and the deep gray matter following hyperoxia (41, 42). Of note, we also detected an increased neuronal cell death in parts of the hippocampus (i.e., CA1 and CA2) which seems to contrast a previous report revealing no impact of hyperoxia on cellular degeneration in the dentate gyrus (42). This difference might be explained by the different substructures analyzed in the former study and by the fact that the dentate gyrus belongs to the archicortex which respond different to cellular stress compared with the neocortex (43). Furthermore, in contrast to the previous study, we performed cell-specific analysis by NeuN/ TUNEL co-staining to specifically analyze neuronal degeneration which is limited with a single TUNEL staining. Even though the importance of Ras in cell survival is widely accepted, for specific pathophysiological cases and cellular systems, it has been shown that Ras may also promote cell death (44). In this study, there are no indications for such detrimental effects probably due to developmental differences, i.e., transgenic synRas mice exposed to normoxia did not show an increased cellular degeneration compared with wild-type littermate controls. Therefore, the immature brain might be less sensitive to proapoptotic effects of Ras activation and thus potential harmful effects.
Surprisingly, in addition to protection from neuronal death, we observed a marked decrease in hyperoxia-induced oligodendrocyte degeneration in synRas-transgenic mice. Since in these mice, Ras activity is only enhanced in neurons, an indirect impact of the neuronal compartment on oligodendrocyte responses to hyperoxia can be assumed. These results clearly support the current concept of an intense communication between neurons/axons and oligodendrocytes/myelin $(31,33)$. Interestingly, elevated Ras signaling exclusively in oligodendrocytes has been shown to mediate opposite effects (45). Increased Ras activity in oligodendrocytes of healthy adult mice resulted in myelin decompaction (45) emphasizing the importance of cell-specific analysis and the divergent roles of Ras-mediated signaling dependent on the pathophysiological context, the cellular system, and developmental stage. Nevertheless, expression was restricted to mature oligodendrocytes. Novel transgenic mouse models directly targeting immature oligodendrocyte precursor cells (OPCs) would help to clearly define the interplay between neuronal responses to hyperoxia and developmental processes of myelination in neonatal subjects.

Protective effects on acute oligodendrocyte degeneration were accompanied by improved myelination 4 days after hyperoxia. 
Interestingly, no differences were observed for the abundance of Olig2 at this time point, indicating compensatory oligodendrocyte proliferation, which, however fails to restore myelination deficits (37). This might be well explained by impaired oligodendrocyte maturation and differentiation following hyperoxia as previously described $(21,37)$. Increased MBP expression at similar Olig2 levels in hyperoxic syn Ras suggests that neuronal Ras activation positively influences differentiation capacity of OPCs in the context of neonatal oxygen-induced toxicity.

In addition to subacute effects on oligodendrocyte survival and differentiation, neonatal hyperoxia causes long-lasting structural alterations on tensor imaging scans of adult brains, which is accompanied by significant cognitive deficits (21). In this study, we provide more detailed information about axon-myelin integrity with ultrastructural analysis of myelin sheaths in young adult animals exposed to neonatal hyperoxia. Here, we detected a considerable amount of non-myelinated axons in hyperoxic wild-type mice, which was significantly improved in synRas mice. Furthermore, the percentage of myelinated axons with abnormal myelin structures was significantly lower in synRas mice. While being in concordance with previous descriptions of hyperoxiarelated disturbed ultrastructural myelin integrity (18), our results add important new knowledge, because pathological alterations of the WM were improved solely by modulation of the neuronal compartment, represented by increased Ras activity.

Different mechanisms may account for our observations. First, according to the initial description by Heumann et al. synRas mice reveal an increased expression of neuropeptide Y (29), which has been shown to improve myelination in the neonatal brain via induction of neurotrophin 3 (46). Second, increased neuronal activity described for $\operatorname{syn}$ Ras mice displayed by enhanced glutamatergic transmission, and long-term potentiation (30) may explain our findings. Whereas the essential need of intact myelination for preservation and maintenance of axonal structure and function is without any doubt (47), there is compelling evidence for a substantial communication into the other direction, i.e., an activity-dependent signaling from neurons/ axons to oligodendrocytes/myelin (33). Accordingly, recent studies indicate that axonal action potentials activate myelinic NMDA receptors (48) resulting in impaired metabolic coupling between axons and oligodendrocytes (49). In addition to metabolic processes, neuronal activity is supposed to determine the release of BDNF (50), a growth factor particularly important for OPC development and maturation $(51,52)$. While much focus has been given to neuronal activity affecting OPC responses during physiological development, potential interactions in response to pathology in the developing brain are less explored. Considering the aforementioned studies, we speculate that hyperoxia modulates neuronal/axonal function and activity due to reduced Ras activity, which may be compensated by neuronal Ras overactivation thereby improving oligodendrocyte differentiation and long-term myelination capacity. A clear goal for future work will be to characterize neuronal/axonal function and activity in hyperoxic animals with constitutively expressed activated Ras in the neuronal compartment. This is further supported by a very recent report that the pattern of neuronal activity triggers distinct responses of OPC proliferation and differentiation (53).
Strengths of this study are well statistically powered analyses, long-term evaluation of myelination deficits, and the use of a cellspecific transgenic mouse model. A potential limitation might be that this mouse model did not allow for conditional transgene expression initiated at various time points. Nevertheless, as in synRas mice, constitutive Ras activation starts at postnatal day 4, physiological effects on embryonic neuronal development can be excluded. Furthermore, long-term analyses of neurodevelopmental behavior and of axonal function/integrity would have strengthened our hypothesis. However, previous experimental studies combined with clinical data provide clear evidence for a good correlation between alterations of WM development and long-term behavioral deficits as well as axonal integrity and function $(14,18-21,39)$.

To conclude, this work demonstrated that hyperoxia-induced impairment of neurodevelopment does not solely rely on direct modulation of oligodendrocyte responses but is also affected by neuronal cell signaling with major impact on WM development. This work emphasizes the unmet need for cell-specific analysis in models of neonatal brain injury to identify more specific targets for therapeutic intervention.

\section{ETHICS STATEMENT}

All animal experiments were approved and performed in accordance with the guidelines of the University Hospital Essen, Germany and with local government approval by the State Agency for Nature, Environment and Consumer Protection North Rhine-Westphalia.

\section{AUTHOR CONTRIBUTIONS}

MS, IB, JH, KK, RH, and HJ designed and performed the experiments and analyzed the data. MS, JH, RH, EW, HJ, UF-M, and IB discussed the data. UF-M and IB initiated and organized the study. MS, JH, UF-M, and IB wrote the manuscript.

\section{ACKNOWLEDGMENTS}

The authors thank Dorothea Schünke for her ambitious help and patience preparing sections for electron microscopy and Mandana Rizazad for ongoing excellent technical assistance.

\section{FUNDING}

This work was supported by the Mercator Research Center Ruhr (\# Pr-2011-0066, to RH, UF-M, IB), Karl-Heinz Frenzen- and C. D.-Stiftung (to JH, UF-M, IB). RH was funded by HORIZON 2020, No 686841. Furthermore, we acknowledge support by the Open Access Publication Fund of the University of Duisburg-Essen.

\section{SUPPLEMENTARY MATERIAL}

The Supplementary Material for this article can be found online at https://www.frontiersin.org/articles/10.3389/fneur.2018.00175/ full\#supplementary-material. 


\section{REFERENCES}

1. Kusuda S, Fujimura M, Uchiyama A, Totsu S, Matsunami K, Neonatal Research Network Japan. Trends in morbidity and mortality among very-lowbirth-weight infants from 2003 to 2008 in Japan. Pediatr Res (2012) 72:531-8. doi:10.1038/pr.2012.114

2. Stoll BJ, Hansen NI, Bell EF, Shankaran S, Laptook AR, Walsh MC, et al. Neonatal outcomes of extremely preterm infants from the NICHD neonatal research network. Pediatrics (2010) 126:443-56. doi:10.1542/peds.2009-2959

3. Keller M, Felderhoff-Mueser U, Lagercrantz H, Dammann O, Marlow N, Huppi P, et al. Policy benchmarking report on neonatal health and social policies in 13 European countries. Acta Paediatr (2010) 99:1624-9. doi:10.1111/j.1651-2227.2010.01894.x

4. Ananth CV, Joseph KS, Oyelese Y, Demissie K, Vintzileos AM. Trends in preterm birth and perinatal mortality among singletons: United States, 1989 through 2000. Obstet Gynecol (2005) 105:1084-91. doi:10.1097/01. AOG.0000158124.96300.c7

5. Oskoui M, Coutinho F, Dykeman J, Jette N, Pringsheim T. An update on the prevalence of cerebral palsy: a systematic review and meta-analysis. Dev Med Child Neurol (2013) 55:509-19. doi:10.1111/dmcn.12080

6. Woodward LJ, Anderson PJ, Austin NC, Howard K, Inder TE. Neonatal MRI to predict neurodevelopmental outcomes in preterm infants. $N$ Engl J Med (2006) 355:685-94. doi:10.1056/NEJMoa053792

7. Thompson DK, Wood SJ, Doyle LW, Warfield SK, Lodygensky GA, Anderson PJ, et al. Neonate hippocampal volumes: prematurity, perinatal predictors, and 2-year outcome. Ann Neurol (2008) 63:642-51. doi:10.1002/ ana. 21367

8. Doyle LW, Anderson PJ. Adult outcome of extremely preterm infants. Pediatrics (2010) 126:342-51. doi:10.1542/peds.2010-0710

9. Monson BB, Anderson PJ, Matthews LG, Neil JJ, Kapur K, Cheong JL, et al. Examination of the pattern of growth of cerebral tissue volumes from hospital discharge to early childhood in very preterm infants. JAMA Pediatr (2016) 170:772-9. doi:10.1001/jamapediatrics.2016.0781

10. Shah PK, Prabhu V, Karandikar SS, Ranjan R, Narendran V, Kalpana N. Retinopathy of prematurity: past, present and future. World J Clin Pediatr (2016) 5:35-46. doi:10.5409/wjcp.v5.i1.35

11. Day CL, Ryan RM. Bronchopulmonary dysplasia: new becomes old again! Pediatr Res (2017) 81:210-3. doi:10.1038/pr.2016.201

12. Collins MP, Lorenz JM, Jetton JR, Paneth N. Hypocapnia and other ventilation-related risk factors for cerebral palsy in low birth weight infants. Pediatr Res (2001) 50:712-9. doi:10.1203/00006450-200112000-00014

13. Deulofeut R, Dudell G, Sola A. Treatment-by-gender effect when aiming to avoid hyperoxia in preterm infants in the NICU. Acta Paediatr (2007) 96:990-4. doi:10.1111/j.1651-2227.2007.00365.x

14. Back SA, Rosenberg PA. Pathophysiology of glia in perinatal white matter injury. Glia (2014) 62:1790-815. doi:10.1002/glia.22658

15. Gerstner B, Desilva TM, Genz K, Armstrong A, Brehmer F, Neve RL, et al. Hyperoxia causes maturation-dependent cell death in the developing white matter. J Neurosci (2008) 28:1236-45. doi:10.1523/JNEUROSCI.3213-07.2008

16. Vottier G, Pham H, Pansiot J, Biran V, Gressens P, Charriaut-Marlangue C, et al. Deleterious effect of hyperoxia at birth on white matter damage in the newborn rat. Dev Neurosci (2011) 33:261-9. doi:10.1159/000327245

17. Brehmer F, Bendix I, Prager S, Van De Looij Y, Reinboth BS, Zimmermanns J, et al. Interaction of inflammation and hyperoxia in a rat model of neonatal white matter damage. PLoS One (2012) 7:e49023. doi:10.1371/journal. pone.0049023

18. Ritter J, Schmitz T, Chew LJ, Buhrer C, Mobius W, Zonouzi M, et al. Neonatal hyperoxia exposure disrupts axon-oligodendrocyte integrity in the subcortical white matter. J Neurosci (2013) 33:8990-9002. doi:10.1523/ JNEUROSCI.5528-12.2013

19. Hoeber D, Sifringer M, Van De Looij Y, Herz J, Sizonenko SV, Kempe K, et al. Erythropoietin restores long-term neurocognitive function involving mechanisms of neuronal plasticity in a model of hyperoxia-induced preterm brain injury. Oxid Med Cell Longev (2016) 2016:9247493. doi:10.1155/2016/9247493

20. Schmitz T, Endesfelder S, Reinert MC, Klinker F, Muller S, Buhrer C, et al. Adolescent hyperactivity and impaired coordination after neonatal hyperoxia. Exp Neurol (2012) 235:374-9. doi:10.1016/j.expneurol.2012.03.002
21. Serdar M, Herz J, Kempe K, Lumpe K, Reinboth BS, Sizonenko SV, et al. Fingolimod protects against neonatal white matter damage and long-term cognitive deficits caused by hyperoxia. Brain Behav Immun (2016) 52:106-19. doi:10.1016/j.bbi.2015.10.004

22. Gerstner B, Sifringer M, Dzietko M, Schuller A, Lee J, Simons S, et al. Estradiol attenuates hyperoxia-induced cell death in the developing white matter. Ann Neurol (2007) 61:562-73. doi:10.1002/ana.21118

23. Sifringer M, Genz K, Brait D, Brehmer F, Lober R, Weichelt U, et al. Erythropoietin attenuates hyperoxia-induced cell death by modulation of inflammatory mediators and matrix metalloproteinases. Dev Neurosci (2009) 31:394-402. doi:10.1159/000232557

24. Bendix I, Schulze C, Haefen C, Gellhaus A, Endesfelder S, Heumann R, et al. Erythropoietin modulates autophagy signaling in the developing rat brain in an in vivo model of oxygen-toxicity. Int J Mol Sci (2012) 13:12939-51. doi:10.3390/ijms131012939

25. Bendix I, Weichelt U, Strasser K, Serdar M, Endesfelder S, Von Haefen C, et al. Hyperoxia changes the balance of the thioredoxin/peroxiredoxin system in the neonatal rat brain. Brain Res (2012) 1484:68-75. doi:10.1016/ j.brainres.2012.09.024

26. Sifringer M, Bendix I, Borner C, Endesfelder S, Von Haefen C, Kalb A, et al. Prevention of neonatal oxygen-induced brain damage by reduction of intrinsic apoptosis. Cell Death Dis (2012) 3:e250. doi:10.1038/cddis.2011.133

27. Felderhoff-Mueser U, Bittigau P, Sifringer M, Jarosz B, Korobowicz E, Mahler L, et al. Oxygen causes cell death in the developing brain. Neurobiol Dis (2004) 17:273-82. doi:10.1016/j.nbd.2004.07.019

28. Manns M, Leske O, Gottfried S, Bichler Z, Lafenetre P, Wahle P, et al. Role of neuronal ras activity in adult hippocampal neurogenesis and cognition. Front Neurosci (2011) 5:18. doi:10.3389/fnins.2011.00018

29. Heumann R, Goemans C, Bartsch D, Lingenhohl K, Waldmeier PC, Hengerer B, et al. Transgenic activation of Ras in neurons promotes hypertrophy and protects from lesion-induced degeneration. J Cell Biol (2000) 151:1537-48. doi:10.1083/jcb.151.7.1537

30. Arendt T, Gartner U, Seeger G, Barmashenko G, Palm K, Mittmann T, et al. Neuronal activation of Ras regulates synaptic connectivity. Eur J Neurosci (2004) 19:2953-66. doi:10.1111/j.0953-816X.2004.03409.x

31. Nave KA, Trapp BD. Axon-glial signaling and the glial support of axon function. Annu Rev Neurosci (2008) 31:535-61. doi:10.1146/annurev.neuro. 30.051606.094309

32. Zuchero JB, Barres BA. Intrinsic and extrinsic control of oligodendrocyte development. Curr Opin Neurobiol (2013) 23:914-20. doi:10.1016/j.conb. 2013.06.005

33. Micu I, Plemel JR, Caprariello AV, Nave KA, Stys PK. Axo-myelinic neurotransmission: a novel mode of cell signalling in the central nervous system. Nat Rev Neurosci (2017) 19(1):58. doi:10.1038/nrn.2017.166

34. Dobbing J, Sands J. Comparative aspects of the brain growth spurt. Early Hum Dev (1979) 3:79-83. doi:10.1016/0378-3782(79)90022-7

35. Craig A, Ling Luo N, Beardsley DJ, Wingate-Pearse N, Walker DW, Hohimer AR, et al. Quantitative analysis of perinatal rodent oligodendrocyte lineage progression and its correlation with human. Exp Neurol (2003) 181:231-40. doi:10.1016/S0014-4886(03)00032-3

36. Semple BD, Blomgren K, Gimlin K, Ferriero DM, Noble-Haeusslein LJ. Brain development in rodents and humans: identifying benchmarks of maturation and vulnerability to injury across species. Prog Neurobiol (2013) 10(6-107):1-16. doi:10.1016/j.pneurobio.2013.04.001

37. Schmitz T, Ritter J, Mueller S, Felderhoff-Mueser U, Chew LJ, Gallo V. Cellular changes underlying hyperoxia-induced delay of white matter development. J Neurosci (2011) 31:4327-44. doi:10.1523/JNEUROSCI.3942-10.2011

38. Schmitz T, Krabbe G, Weikert G, Scheuer T, Matheus F, Wang Y, et al. Minocycline protects the immature white matter against hyperoxia. Exp Neurol (2014) 254:153-65. doi:10.1016/j.expneurol.2014.01.017

39. Reich B, Hoeber D, Bendix I, Felderhoff-Mueser U. Hyperoxia and the immature brain. Dev Neurosci (2016) 38:311-30. doi:10.1159/000454917

40. De Hoz L, Simons M. The emerging functions of oligodendrocytes in regulating neuronal network behaviour. Bioessays (2015) 37:60-9. doi:10.1002/ bies.201400127

41. Taglialatela G, Perez-Polo JR, Rassin DK. Induction of apoptosis in the CNS during development by the combination of hyperoxia and inhibition of 
glutathione synthesis. Free Radic Biol Med (1998) 25:936-42. doi:10.1016/ S0891-5849(98)00131-2

42. Endesfelder S, Zaak I, Weichelt U, Buhrer C, Schmitz T. Caffeine protects neuronal cells against injury caused by hyperoxia in the immature brain. Free Radic Biol Med (2014) 67:221-34. doi:10.1016/j.freeradbiomed.2013.09.026

43. Posimo JM, Titler AM, Choi HJ, Unnithan AS, Leak RK. Neocortex and allocortex respond differentially to cellular stress in vitro and aging in vivo. PLoS One (2013) 8:e58596. doi:10.1371/journal.pone.0058596

44. Ehrkamp A, Herrmann C, Stoll R, Heumann R. Ras and rheb signaling in survival and cell death. Cancers (Basel) (2013) 5:639-61. doi:10.3390/ cancers5020639

45. Titus HE, Lopez-Juarez A, Silbak SH, Rizvi TA, Bogard M, Ratner N. Oligodendrocyte RasG12V expressed in its endogenous locus disrupts myelin structure through increased MAPK, nitric oxide, and notch signaling. Glia (2017) 65:1990-2002. doi:10.1002/glia.23209

46. Hashimoto R, Udagawa J, Kagohashi Y, Matsumoto A, Hatta T, Otani $\mathrm{H}$. Direct and indirect effects of neuropeptide $\mathrm{Y}$ and neurotrophin 3 on myelination in the neonatal brains. Brain Res (2011) 1373:55-66. doi:10.1016/ j.brainres.2010.12.027

47. Nave KA, Werner HB. Myelination of the nervous system: mechanisms and functions. Annu Rev Cell Dev Biol (2014) 30:503-33. doi:10.1146/annurevcellbio-100913-013101

48. Micu I, Plemel JR, Lachance C, Proft J, Jansen AJ, Cummins K, et al. The molecular physiology of the axo-myelinic synapse. Exp Neurol (2016) 276: 41-50. doi:10.1016/j.expneurol.2015.10.006

49. Saab AS, Tzvetavona ID, Trevisiol A, Baltan S, Dibaj P, Kusch K, et al. Oligodendroglial NMDA receptors regulate glucose import and axonal energy metabolism. Neuron (2016) 91:119-32. doi:10.1016/j.neuron.2016. 05.016
50. Ghosal S, Bang E, Yue W, Hare BD, Lepack AE, Girgenti MJ, et al. Activitydependent brain-derived neurotrophic factor release is required for the rapid antidepressant actions of scopolamine. Biol Psychiatry (2018) 83:29-37. doi:10.1016/j.biopsych.2017.06.017

51. Xiao J, Wong AW, Willingham MM, Van Den Buuse M, Kilpatrick TJ, Murray SS. Brain-derived neurotrophic factor promotes central nervous system myelination via a direct effect upon oligodendrocytes. Neurosignals (2010) 18:186-202. doi:10.1159/000323170

52. Ramos-Cejudo J, Gutierrez-Fernandez M, Otero-Ortega L, Rodriguez-Frutos B, Fuentes B, Vallejo-Cremades MT, et al. Brain-derived neurotrophic factor administration mediated oligodendrocyte differentiation and myelin formation in subcortical ischemic stroke. Stroke (2015) 46:221-8. doi:10.1161/ STROKEAHA.114.006692

53. Nagy B, Hovhannisyan A, Barzan R, Chen TJ, Kukley M. Different patterns of neuronal activity trigger distinct responses of oligodendrocyte precursor cells in the corpus callosum. PLoS Biol (2017) 15:e2001993. doi:10.1371/ journal.pbio. 2001993

Conflict of Interest Statement: The authors declare that the research was conducted in the absence of any commercial or financial relationships that could be construed as a potential conflict of interest.

Copyright $\odot 2018$ Serdar, Herz, Kempe, Winterhager, Jastrow, Heumann, FelderhoffMüser and Bendix. This is an open-access article distributed under the terms of the Creative Commons Attribution License (CC BY). The use, distribution or reproduction in other forums is permitted, provided the original author(s) and the copyright owner are credited and that the original publication in this journal is cited, in accordance with accepted academic practice. No use, distribution or reproduction is permitted which does not comply with these terms. 